\title{
Autopercepção das condições de saúde bucal de idosos do município de Araraquara - SP
}

\section{Self-perception of oral health status by elderly in Araraquara - SP city}

\section{Cristiane HENRIQUES}

Doutoranda - Programa de Pós-Graduação em Odontologia - Área de Concentração em Dentística Restauradora Faculdade de Odontologia de Araraquara - Universidade Estadual Paulista - UNESP - Araraquara - SP - Brasil

\section{Rodolpho TELAROLLI JÚNIOR}

Professor Adjunto - Departamento de Ciências Biológicas - Faculdade de Ciências Farmacêuticas de Araraquara Universidade Estadual Paulista - UNESP - Araraquara - SP - Brasil

\section{Leonor de Castro Monteiro LOFFREDO}

Professora Adjunta - Departamento de Odontologia Social - Faculdade de Odontologia de Araraquara - Universidade Estadual Paulista - UNESP - Araraquara - SP - Brasil

\section{Andréia Affonso Barreto MONTANDON Juliana Alvares Duarte Bonini CAMPOS}

Professora Assistente Doutora - Departamento de Odontologia Social - Faculdade de Odontologia de Araraquara Universidade Estadual Paulista - UNESP - Araraquara - SP - Brasil

\begin{abstract}
Resumo
O objetivo deste estudo foi verificar a saúde bucal e a autopercepção de 61 idosos atendidos na FOAR/UNESP, no ano de 2001. Para avaliação da autopercepção utilizou-se o Geriatric Oral Health Assessment Index (GOHAI) e o questionário proposto por Silva \& Castellanos Fernandes14 (2001) (S\&CF). Um examinador realizou exame clínico e adotou-se (0) para ausência e (1) para presença de problemas periodontais ou cárie radicular e, quando da presença de próteses $(0)$ para não funcionais e (1) para funcionais. Realizou-se análise descritiva das variáveis. Testou-se a reprodutibilidade

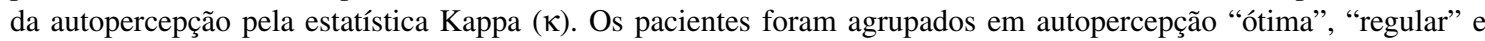
"ruim" e a associação com doença periodontal, cárie radicular e uso de prótese foi realizada pelo teste de qui-quadrado ou teste exato de Fisher, com significância de 5\%. A maioria dos idosos afirmou nunca ter sofrido limitações devido a seus dentes, gengivas ou próteses, entretanto, o Índice GOHAI global foi de 27,77, característico de uma autopercepção ruim. Observou-se que 56\% dos idosos apresentaram doença periodontal, 38\% cárie radicular e $92 \%$ dos idosos eram usuários de próteses não funcionais. Houve baixa concordância entre os diferentes métodos de avaliação $(\kappa=0,087) \mathrm{e}$ associação não-significativa entre o índice GOHAI e doença periodontal $(\chi 2=0,856 ; p=0,5133)$, cárie radicular $(\chi 2=0,002$; p=0,9636) e o uso de próteses (Exato de Fisher: p=0,3286). As condições de saúde bucal apresentaram-se insatisfatórias e a autopercepção mostrou-se ruim, com associação não-significativa com as variáveis clínicas doença periodontal, cárie radicular e uso de prótese.
\end{abstract}

\section{Palavras-Chave}

Autopercepção; idoso; saúde bucal. 


\section{INTRODUÇÃO}

O aumento da expectativa de vida dos brasileiros vem ocorrendo de forma crescente e caracteriza-se por um processo de transição demográfica importante (Stenberg \& Gordon ${ }^{15}$, 1998; Veras ${ }^{16}, 2003$; Silva et al. $\left.{ }^{13}, 2005\right)$. Esse envelhecimento da população tem despertado interesse para as peculiaridades dessa faixa etária, atentando para suas necessidades nos aspectos de saúde, sociais e econômicos.

No campo da saúde, este processo alterou padrões de morbi/mortalidade, com elevação de doenças crônico-degenerativas, levando a uma transição epidemiológica (Berquó \& Leite ${ }^{3}$, 1988).

Em relação às condições de saúde bucal, dados apresentados pelo Levantamento das Condições de Saúde Bucal da População Brasileira no ano 2000 (Ministério da Saúde ${ }^{4}, 2004$ ) apontam sua deterioração com o transcorrer da idade em todos os aspectos considerados.

Segundo Silva \& Castellanos Fernandes ${ }^{14}$ (2001), o Brasil vive um contexto de abandono e dificuldades, que poderia ser minimizado se houvessem ações educativas voltadas para autoproteção, conscientizando a comunidade para a necessidade de cuidados com a saúde bucal. Um aspecto importante a ser considerado é o da autopercepção, onde as atitudes individuais poderão levar à mudança de comportamento de uma comunidade, de forma que indicadores desta autopercepção se constituam em importante ferramenta para a implantação de serviços odontológicos voltados para esta camada populacional.

Os dados sobre autopercepção são subjetivos e para sua coleta, alguns autores padronizaram questionários para avaliar os problemas funcionais, sociais e psicológicos decorrentes das doenças bucais. Dentre estes, destaca-se o empregado para obtenção do índice GOHAI - Geriatric Oral Health Assessment Index desenvolvido por Atchinson \& Dolan ${ }^{1}$ (1990). Outro questionário bastante simples de aplicação se restringe a autoavaliação de saúde bucal e de problemas nos dentes, gengivas e próteses, proposto por Silva \& Castellanos Fernandes ${ }^{14}$ (2001) - S\&CF.

A exemplo dos dois questionários citados acima, existem outros instrumentos elaborados para complementar os indicadores clínicos de saúde bucal e são de utilidade para obtenção de informações que possam ser utilizadas em programas educativos, preventivos e curativos. ${ }^{10}$

Dada a relevância de se conhecer o estado de saúde bucal de idosos que demandaram atendimento junto à Clínica da FOAr/UNESP, bem como sua autopercepção, procedeu-se a este estudo, com o objetivo de fornecer subsídios para o atendimento odontológico de idosos, aplicando-se o índice GOHAI, o modelo de S\&CF e exame clínico.

\section{Material e mÉtodo}

O delineamento metodológico foi de um estudo de corte transversal. A amostra foi constituída por 61 pacientes, que demandaram atendimento junto à Clínica de Prótese Parcial Removível da Faculdade de Odontologia de Araraquara - UNESP, no ano de 2001, tendo sido adotada a técnica de amostragem por conveniência.

Como critério de inclusão adotou-se idade mínima de 60 anos, com pelo menos dois dentes presentes por hemiarco da cavidade bucal e funcionalmente capazes, de acordo com os critérios de Rosa et al. ${ }^{12}$ (2003), onde estão incluídos aqueles indivíduos que podem apresentar doenças crônicas não graves e controladas por medicação ou que apresentam declínio sensorial associado à idade, mas que vivem sem necessitar de ajuda. 
Para avaliação da autopercepção dos idosos em relação à saúde bucal utilizou-se como instrumento de medida os questionários GOHAI (Quadro 1) e S\&CF (Quadro 2).

Analisando o Quadro 1, podem ser observados os doze itens que compõem o índice GOHAI, se constituindo em informações a respeito das influências dos problemas de saúde bucal em três dimensões, física, psicológica e dor ou desconforto. O nível de mensuração destes itens é categórico de forma que às respostas "sempre", "às vezes" e "nunca" foram atribuídos pesos 1,2 e 3 respectivamente. Os itens de números 3,5 e 7 são computados inversamente aos demais. Para obtenção do índice global realiza-se a soma simples dos escores, numa escala de 12 a 36 , sendo que o maior valor indica a mais favorável auto-informação a respeito da saúde bucal (Pinto $\left.{ }^{10}, 2000\right)$. De acordo com a Sociedade Americana de Geriatria (Reuben \& Solomon $\left.{ }^{11}, 1989\right)$, nas questões diretas, quanto mais prevalente a categoria "sempre" piores serão as condições de saúde bucal e o contrário para as questões inversas.

O índice GOHAI permite classificar a autopercepção em "ótima" (34 a 36 pontos), "regular" (30 até 33 pontos) e "ruim" (<30 pontos) adaptando-se o critério de Atchison \& Dolan" (1990) para escala simplificada.

Previamente ao levantamento, realizou-se um plano piloto onde foram entrevistados 10 pacientes por um único examinador, com experiência em aplicação dos questionários, tendo sido obtida concordância intra-examinador de $100 \%$ nas respostas. Assim, esse examinador, com experiência em entrevistas, realizou exame clínico em uma cadeira odontológica sob luz de refletor, após profilaxia dental com o auxílio de sonda exploradora número 5, espelho bucal plano e sonda periodontal desenvolvida pela OMS (Organização Mundial de Saúde).

Os dados do exame clínico foram anotados em ficha pré-codificada, adotando-se (0) para ausência e (1) para presença de problemas periodontais ou cárie radicular e, quando da presença de próteses adotouse (0) para não funcionais e (1) para funcionais. Classificaram-se como próteses funcionais as que preenchessem os requisitos clínicos de estabilidade, adaptação, retenção e integridade; caso pelo menos um desses requisitos estivesse comprometido, as próteses foram consideradas como não funcionais.

O planejamento estatístico compreendeu as fases:

- Inicialmente, estimou-se a reprodutibilidade da autopercepção dos idosos segundo os questionários referentes ao GOHAI e S\&CF aplicando-se a estatística Kappa ( $\kappa)$, e sua classificação segundo os padrões de Landis \& Koch7 (1977).

- As variáveis de estudo referentes a autopercepção da saúde bucal e a problemas de saúde bucal, tais como doença periodontal, cárie radicular e utilização de próteses foram estudadas mediante análise descritiva dos dados obtidos utilizando o "software" Excel.

- Os pacientes foram agrupados segundo o índice GOHAI em autopercepção "ótima", "regular" e "ruim"e sua associação com o diagnóstico clínico de doença periodontal, cárie radicular e uso de próteses funcionais foi estudada por meio do teste de qui-quadrado ou teste exato de Fisher, ao nível de significância de 5\%.

Deve-se ressaltar que este estudo foi aprovado pelo Comitê de Ética em Pesquisa da FOAr/UNESP sob o protocolo $\mathrm{n}^{\circ} 46 / 00$.

\section{Resultados}

As freqüências relativas (\%) dos componentes do GOHAI podem ser observadas na Tabela 1 .

Chama a atenção, na Tabela 1 , que a maioria dos idosos afirmou nunca ter sofrido limitações (físicas, psicológicas, dor ou desconforto) devido a seus dentes ou próteses. Ainda, 78,7\% relataram estarem felizes com o aspecto de seus dentes ou prótese. Entretanto, $75,4 \%$ sentiram desconforto ao engolir o alimento.

O Índice GOHAI global calculado para estes idosos foi de 27,77, com desvio-padrão de 3,74. Ainda que a estimativa por ponto do índice $(27,77)$ denote um nível ruim de auto-percepção, a estimativa por intervalo de confiança $(20,44$ - 35,10) faz com que autopercepção atinja nível regular.

Quando os idosos foram questionados sobre sua percepção da presença de problemas na gengiva, dentes ou próteses, observou-se, segundo o questionário S\&CF, que 56\% afirmaram não apresentar estes problemas, enquanto $8 \%$ não souberam avaliar e $36 \%$ reconheceram ter esses problemas; adicionalmente, $42,6 \%$ mostraram-se preocupados com este aspecto abordado no item 9 do índice GOHAI.

Além disso, no questionário $\mathrm{S} \& \mathrm{CF}$, a maioria dos entrevistados estava satisfeita com sua condição de saúde bucal. Entretanto, 44\% das pessoas reconheceram problemas com sua saúde bucal ou não conseguiram dimensioná-los. 
Tabela 1. Distribuição de freqüência (\%) das respostas dadas pelos idosos segundo cada questão componente do índice GOHAl. Araraquara, 2001.

\begin{tabular}{|c|c|c|c|}
\hline Componentes - Índice GOHAI & & $\%$ & \\
\hline Nos últimos três meses... & Sempre & às vezes & nunca \\
\hline $\begin{array}{l}1 \text { - Limitou o tipo ou a quantidade de alimentos que come devido a problemas com } \\
\text { seus dentes ou próteses? }\end{array}$ & 13,1 & 21,3 & 65,6 \\
\hline $\begin{array}{l}2 \text { - Tem problemas mordendo ou mastigando alimentos como carne sólida ou } \\
\text { maçã? }\end{array}$ & 19,7 & 27,9 & 52,5 \\
\hline *3 - Foi capaz de engolir confortavelmente? & 4,9 & 19,7 & 75,4 \\
\hline 4 - Seus dentes ou prótese(s) o impediram de falar da maneira como queriam? & 8,2 & 29,5 & 62,3 \\
\hline${ }^{\star} 5$ - Foi capaz de comer qualquer coisa sem sentir desconforto? & 57,4 & 32,8 & 9,8 \\
\hline $\begin{array}{l}6 \text { - Limitou seus contatos com outras pessoas devido às condições de seus dentes } \\
\text { ou próteses? }\end{array}$ & 0,0 & 8,2 & 91,8 \\
\hline${ }^{\star} 7$ - Sentiu-se contente ou feliz com o aspecto de seus dentes ou próteses? & 50,8 & 27,9 & 21,3 \\
\hline 8 - Usou medicamentos para avaliar dor ou desconforto relativos à boca? & 0,0 & 4,9 & 95,1 \\
\hline 9 - Preocupou-se ou teve cuidados com seus dentes, gengivas ou próteses? & 34,4 & 8,2 & 57,4 \\
\hline $\begin{array}{l}10 \text { - Sentiu-se nervoso ou tomou consciência de problemas com seus dentes, } \\
\text { gengivas ou próteses? }\end{array}$ & 34,4 & 8,2 & 57,4 \\
\hline $\begin{array}{l}11 \text { - Sentiu desconforto ao alimentar-se em frente a outras pessoas devido a } \\
\text { problema com seus dentes ou próteses? }\end{array}$ & 34,4 & 8,2 & 57,4 \\
\hline $\begin{array}{l}12 \text { - Teve sensibilidade nos dentes ou gengivas ao contato com calor, frio ou } \\
\text { doces? }\end{array}$ & 1,6 & 26,2 & 72,1 \\
\hline
\end{tabular}

*perguntas indiretas

A condição de saúde bucal foi aferida no exame clínico e os resultados constam do Gráfico 2.

Observa-se que $56 \%$ dos idosos apresentaram doença periodontal e 38\% cárie radicular, sendo que $25 \%$ dos idosos apresentaram doença periodontal e cárie radicular.

Gráfico 2. Distribuição de idosos segundo a presença de doença periodontal e/ou cárie dental. Araraquara, 2001.

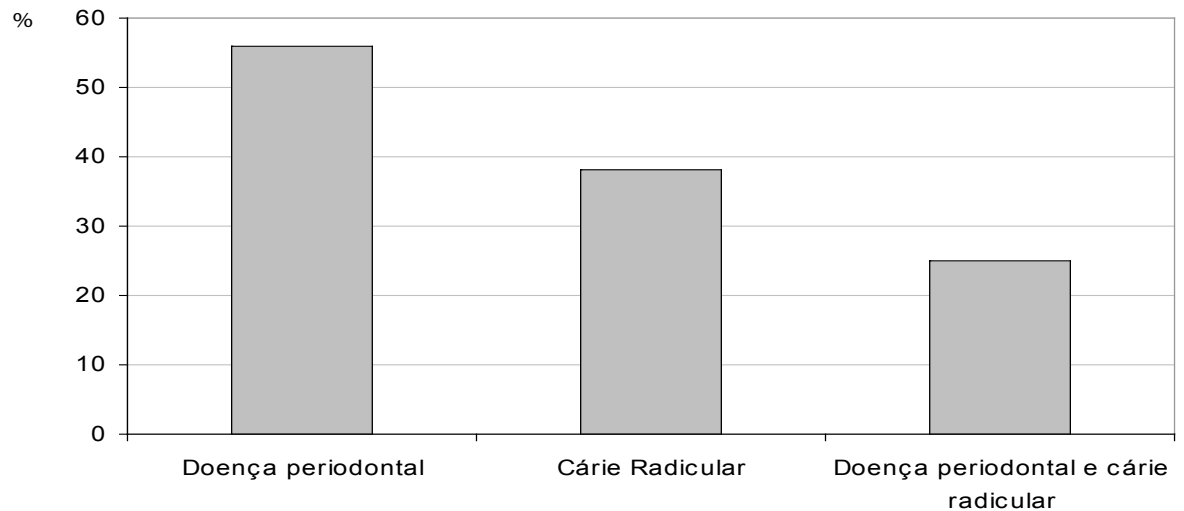


Convém acrescentar que, entre os idosos, $92 \%$ eram usuários de próteses não-funcionais.

A concordância da classificação da autopercepção nas categorias ruim, regular e ótima foi analisada segundo as escalas adotadas, GOHAI e S\&CF.

Pela observação da Tabela 2 pode-se notar que houve baixa concordância entre os diferentes métodos de avaliação da autopercepção $(\kappa=0,087)$, refletindo reprodutibilidade fraca.

A associação entre o índice GOHAI e a doença periodontal aferida pelo exame clínico foi avaliada (Tabela 3).

Para o estudo da associação entre o índice GOHAI e cárie radicular avaliada no exame clínico, os resultados constam na Tabela 4.

Para o estudo da associação entre o índice GOHAI e cárie radicular, verificou-se associação nãosignificativa $\left(\chi^{2}=0,002 ; \mathrm{p}=0,9636\right)$.

$\mathrm{Na}$ Tabela 5 encontram-se os dados do estudo de associação entre o índice GOHAI e o uso de próteses avaliadas por exame clínico.

Pode-se notar que, a associação entre o índice GOHAI e a funcionalidade da prótese foi não-significativa (teste Exato de Fisher: $\mathrm{p}=0,3286$ ) (Tabela 5).

Frente ao exposto, pode-se afirmar que houve associação não-significativa entre a autopercepção dos idosos e a condição de saúde bucal dos mesmos segundo avaliação clínica.

\section{Discussão}

Este estudo teve planejamento metodológico do tipo transversal, sendo adotado o processo de amostra-

\section{Tabela 2. Concordância na classificação da auto- percepção dos idosos: GOHAl e S\&CF Araraquara, 2001}

\begin{tabular}{|c|c|c|c|c|}
\hline \multirow[t]{2}{*}{$\begin{array}{l}\text { Índice } \\
\text { GOHAI }\end{array}$} & \multicolumn{3}{|c|}{$\begin{array}{c}S \& \text { CF } \\
\text { Como você avalia } \\
\text { a condição de } \\
\text { sua boca? }\end{array}$} & \multirow[t]{2}{*}{ Total } \\
\hline & Ruim & Regular & Ótima & \\
\hline Ruim & 12 & 10 & 15 & 37 \\
\hline Regular & - & 5 & 16 & 21 \\
\hline Ótima & - & - & - & - \\
\hline Total & 12 & 15 & 31 & $58^{\star}$ \\
\hline
\end{tabular}

*Nota: Foram excluídos 3 indivíduos por não saberem classificar sua saúde bucal na questão 1 do questionário $\mathrm{S} \& \mathrm{CF}$. gem por conveniência, delineamento comum a estudos desta natureza, como ressaltado por Colussi \& Freitas ${ }^{5}$ (2002) e Moreira et al. ${ }^{9}$ (2005). Assim, os idosos que demandaram atendimento junto à Clínica de Prótese Parcial Removível da Faculdade de Odontologia de Araraquara no ano de 2001 foram selecionados para compor a amostra, o que poderia representar viés de seleção, podendo se constituir numa limitação do estudo quanto à validade externa.

Tabela 3. Índice GOHAl e doença periodontal segundo exame clínico. Araraquara, 2001.

\begin{tabular}{llll}
\hline & \multicolumn{3}{c}{ Doença periodontal } \\
Índice GOHAI & Presente & Ausente & Total \\
\hline Ruim & 24 & 16 & 40 \\
\hline Regular & 10 & 11 & 21 \\
\hline Total & 34 & 27 & 61 \\
\hline
\end{tabular}

Verificou-se associação não-significativa $\left(\chi^{2}=0,856 ; p=0,5133\right)$.

Tabela 4. Índice GOHAl e cárie radicular segundo exame clínico. Araraquara, 2001

\begin{tabular}{llll}
\hline & \multicolumn{3}{c}{ Cárie radicular } \\
Índice GOHAl & Presente & Ausente & Total \\
\hline Ruim & 15 & 25 & 40 \\
\hline Regular & 8 & 13 & 21 \\
\hline Total & 23 & 38 & 61 \\
\hline
\end{tabular}

Tabela 5. Índice GOHAI segundo uso de próteses funcionais ou não funcionais segundo exame clínico. Araraquara, 2001

\begin{tabular}{llll}
\hline & \multicolumn{3}{c}{ Prótese funcional } \\
Índice GOHAI & Não & Sim & Total \\
\hline Ruim & 38 & 2 & 40 \\
\hline Regular & 18 & 3 & 21 \\
\hline Total & 56 & 5 & 61 \\
\hline
\end{tabular}


Por outro lado, o tema é de suma importância por envolver uma população de idosos, faixa etária que, devido à atual transição demográfica e epidemiológica é geralmente excluída das políticas públicas de saúde bucal no Brasil.

Os idosos apresentaram um valor em relação ao GOHAI característico de uma percepção negativa das condições de saúde bucal, estando este valor abaixo do encontrado por Silva \& Castellanos Fernandes ${ }^{14}$ (2001) em outra população da cidade de Araraquara SP. Em outro estudo, Silva et al. ${ }^{13}$ (2005) encontraram uma média de 33,61 para o índice GOHAI para idosos moradores da cidade de Rio Claro - SP, porém nesta amostra estiveram incluídos tanto indivíduos dentados quanto desdentados, portanto, com delineamento metodológico distinto do estudo atual.

O diagnóstico das condições clínicas mostrou associação não-significativa com a autopercepção (Tabelas 3 a 5), o que pode também ser observado por Mathias et al. ${ }^{8}$ (1995), Jokovic \& $\operatorname{Locker}^{6}(1997)$, Silva \& Castellanos Fernandes ${ }^{14}$ (2001) e Silva et al. ${ }^{13}$ (2005), os quais verificaram que a maioria das pessoas vê sua condição bucal de maneira favorável, mesmo em condições clínicas não satisfatórias, provavelmente porque as medidas clínicas de saúde utilizadas pelo profissional são preditores relativamente fracos da percepção de saúde bucal das pessoas.

O presente estudo vai de encontro com o verificado por Atchinson \& Dolan ${ }^{1}$ (1990), realizado nos Estados Unidos, que relataram associação significativa entre a condição de saúde bucal aferida clinicamente e o índice de GOHAI, sugerindo que a saúde bucal pode ser satisfatoriamente medida pelo próprio paciente.

$\mathrm{O}$ fato dos idosos não terem um julgamento apropriado do estado de sua saúde bucal traz preocupação, pois a autopercepção, como um indicador subjetivo, é um instrumento de avaliação que deve ser considerado, corroborando para a afirmação de Silva \& Castellanos Fernandes ${ }^{14}$ (2001), onde a compreensão do paciente acerca de sua saúde é o primeiro passo na elaboração de um programa que inclua ações educativas, voltadas para o autodiagnóstico e autocuidado, além de ações preventivas e curativas. Por outro lado, o nãoconhecimento da existência de problema de saúde bucal se constitue em barreira ao acesso ao serviço odontológico.

\section{Conclusão}

Na população estudada, condições de saúde bucal apresentaram-se insatisfatórias, o que pode ser evidenciado pelos dados clínicos, com alta prevalência de doença periodontal e próteses não-funcionais. A autopercepção em relação à saúde bucal mostrou-se ruim, com associação não-significativa com as variáveis clínicas referentes à doença periodontal, cárie radicular e uso de próteses não-funcionais.

\section{Abstract}

The aim of this study was to analyse oral health condition and self perceived oral health among 61 elderly people under treatment in the Araraquara Dental School, in 2001. In order to evaluate the perceived oral health, it was used the Geriatric Oral Health Assessment Index (GOHAI) and the Silva \& Castellanos Fernandes14 (2001) questionnaire $(\mathrm{S} \& \mathrm{CF})$. Clinical examination was done by one examiner that classified in absent (0) and presence (1) of periodontal disease or root caries and in functional (1) and non-functional (0) for prosthesis users. It was applied descriptive data analyses. It was estimated the reproducibility of selfperceived oral health by kappa statistics ( $\kappa)$. The patients were classified according "excellent", "regular"and "bad" self perceived oral health and the association between periodontal disease, root caries and prosthesis use was done by chi-square statistics $(\chi 2)$, or exact Fisher's test at $5 \%$ of significance level. The majority of the elderly patients had no limitations caused by their teeth, gingival or prosthesis, but the global GOHAI was 27.77, meaning that they had bad perceived oral health. Indeed, 56\% of them had periodontal disease, $38 \%$ root caries, and $92 \%$ used non-functional prosthesis. It was observed weak agreement between the evaluation methods $(\kappa=0.087)$ and the association was non significant between GOHAI index and periodontal disease $(\chi 2=0.856 ; \mathrm{p}=0.5133)$, root caries $(\chi 2=0.002 ; \mathrm{p}=0.9636)$ and the use of prosthesis (Fisher's exact test: $\mathrm{p}=0.3286$ ). Bad oral health conditions and perceived oral health were observed among these patients, with non-significant association in relationship to clinical parameters such as periodontal disease, root caries and prosthesis users.

\section{KEY-WORDS}

Self concept; aged; oral health. 


\section{Quadro 1 - Perguntas que compõem o índice de determinação da saúde bucal geriátrica (GOHAl), para indivíduos com dentes e edêntulos.}

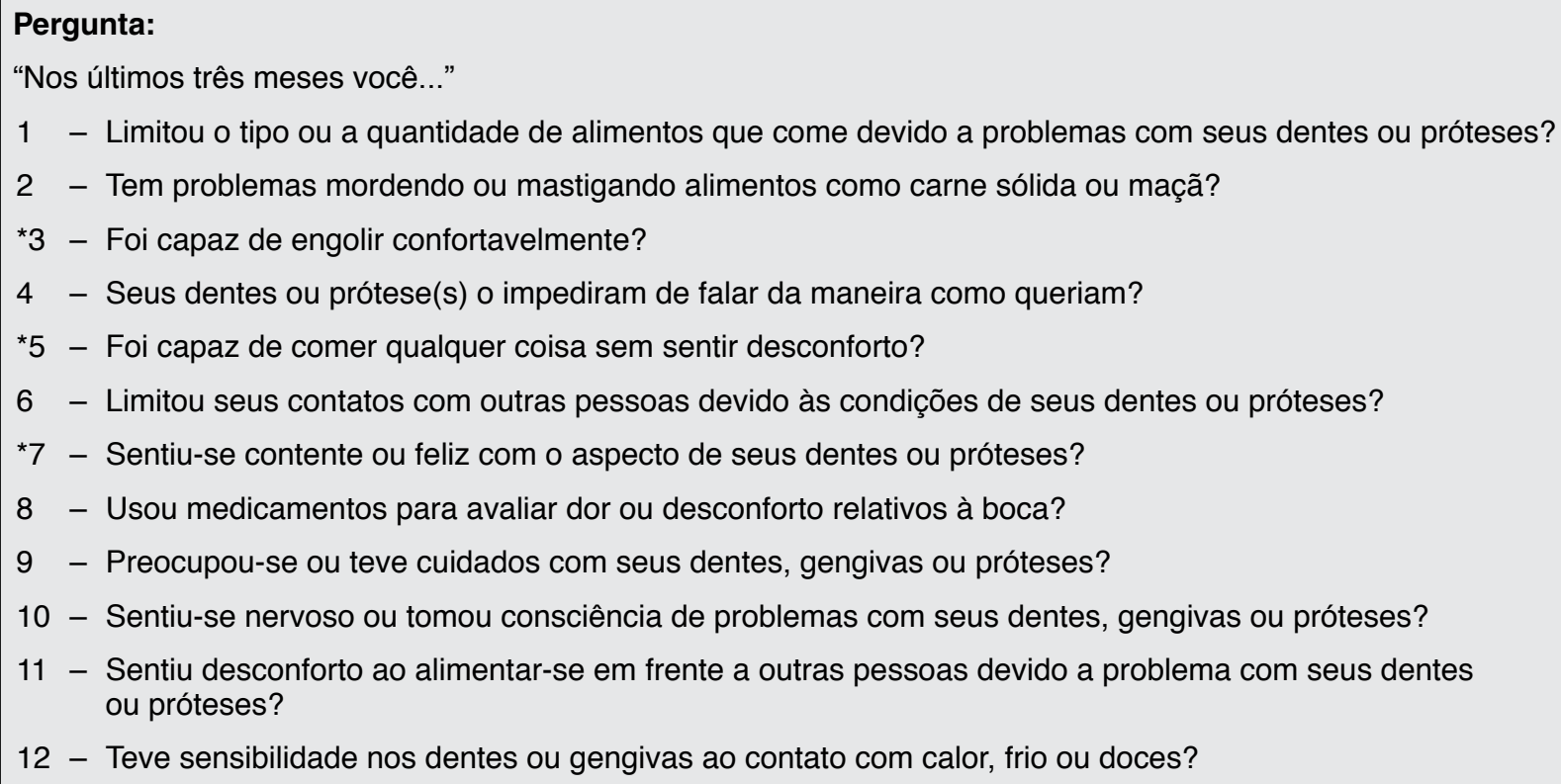

*Contagem inversa em relação à nota máxima que é 3.

Fonte: Atchison 2, 1997.

\section{Quadro 2 - Questões aplicadas para verificar a autopercepção da condição de saúde bucal. Araraquara, 2001.}

1 - Como você avalia a condição de sua boca?
(0) ruim
(1) regular
(2) ótima
(8) não sei

2 - Você tem algum problema com seus dentes, gengiva ou próteses?
(0) Não
(1) Sim
(8) Não sei

\section{REFERÊNCIAS}

1. Atchison KA, Dolan TA. Development of the geriatric oral health assessment index. J Dent Educ. 1990; 54(11): 680-6.

2. Atchison KA. The general oral health assesssment index. In: Slade GD. Measuring oral health and quality of life. North Carolina: University of North Carolina, School of Dentistry. ; 1997. p.71-80.

3. Berquó ES, Leite VM. Algumas considerações sobre a demografia da população idosa no Brasil. Ciênc Cult. 1988; 40(7): 679-88.

4. Brasil. Ministério da Saúde. Projeto SB Brasil 2003: condições de saúde bucal da população brasileira 2002-2003: resultados principais. Brasília: Ministério da Saúde. ; 2004.

5. Colussi CF, Freitas SFT. Aspectos epidemiológicos da saúde bucal do idoso no Brasil. Cad Saúde Pública. 2002 set./out.; 18(5): 1313-20.

6. Jokovic A, Locker D. Dissatisfaction with oral health status in an older adult population. J Public Health Dent. 1997; 57: 40-7.

7. Landis JR, Koch GG. The measurement of observer agreement for categorical data. Biometrics. 1977; 33: 159-74.

8. Mathias RE, Atchison KA, Lubben JE, De Jong F, Scheweitzer SO. Factors affecting self-ratings of oral health. J Public Health Dent. 1995; 55: 197-204.

9. Moreira RS, Nico LS, Tomita NE, Ruiz T. A saúde bucal do idoso brasileiro: revisão sistemática sobre o quadro epidemiológico e acesso aos serviços de saúde bucal. Cad Saúde Pública. 2005 nov./dez.; 21(6): 1665-75.
10. Pinto VG. Identificação de problemas. In: _. Saúde bucal coletiva. São Paulo: Santos; 2000. p.139-222.

11. Reuben DB, Solomon DH. Assessment in geriatrics: of caveats and names. J Am Geriatr Soc. 1989; 37(6):570-2.

12. Rosa TEC, Benício MHD, Latorre MRDO, Ramos LR. Fatores determinantes da capacidade funcional entre idosos. Rev Saúde Pública. 2003; 37(1):40-8.

13. Silva DD, Sousa MLR, Wada RS. Autopercepção e condições de saúde bucal em uma população de idosos. Cad Saúde Pública. 2005 jul./ago.; 21(4):1251-9.

14. Silva SRC, Castellanos Fernandes RA. Autopercepção das condições de saúde bucal por idosos. Rev Saúde Pública. 2001 ago.; 35(4):349-55.

15. Stenberg SA, Gordon M. Who are older adults? Demographics and major health problems. Periodontol 2000. 1998 Feb.; 16: 9-15.

16. Veras R. Em busca de uma assistência adequada à saúde do idoso: revisão da literatura e aplicação de um instrumento de detecção precoce e de previsibilidade de agravos. Cad Saúde Pública. 2003 mai./jun.; 19(3):705-15.

Recebido em: 09/08/06 Aprovado em: 29/08/06

Leonor de Castro Monteiro Loffredo Faculdade de Odontologia de Araraquara - FOAR/UNESP. Departamento de Odontologia Social Rua Humaitá, 1680 - Centro CEP:14801-903 - Araraquara - SP Fone/Fax: (0xx16) 3301-6343 1loffred@foar.unesp.br 